\title{
Comparison of direct plating with the use of enrichment culture for isolation of Aeromonas spp. from faeces
}

\author{
JENNIFER ROBINSON, JANNICE BEAMAN, LINDA WAGENER and VALERIE BURKE
}

Gastroenterology and Nutrition Research Unit, Princess Margaret Children's Medical Research Foundation, Princess Margaret Hospital, GPO Box D184, Perth, Western Australia, 6001

\begin{abstract}
Summary. Direct plating of faecal specimens on blood agar was compared with the use of enrichment culture for isolation of Aeromonas spp. from faeces during a large epidemiological study. Of enterotoxigenic strains isolated by direct plating, $89 \%$ were associated with acute diarrhoea and $7 \%$ with an episode of diarrhoea during the month before collection, but $79 \%$ of enterotoxigenic strains isolated only after enrichment were not associated with acute diarrhoea. With Aeromonas spp., as with intestinal pathogens, it appears that enrichment allows isolation of the bacteria when in low faecal concentrations likely to be found in convalescent patients, carriers and those with subclinical infection. The routine use of enrichment for isolation of faecal aeromonads, by detecting Aeromonas spp. in low numbers in patients without diarrhoea, is likely to confuse interpretation of epidemiological studies seeking to clarify the relationship between Aeromonas spp. and acute diarrhoea.
\end{abstract}

\section{Introduction}

With increased interest in Aeromonas spp. as potential intestinal pathogens, various media for their isolation from faeces have been described (Rogol et al., 1979; Shread et al., 1981; Moulsdale, 1983; Von Graevenitz and Bucher, 1983; Millership and Chattopadhyay, 1984; Robinson et al., 1984). Enrichment culture of specimens in alkaline peptone water has been recommended (Shread et al., 1981; Moulsdale, 1983; Von Graevenitz and Bucher, 1983; Millership and Chattopadhyay, 1984) and has been applied in clinical studies (Echeverria et al., 1981; Millership et al, 1983; Price et al., 1984).

Reluctance to accept Aeromonas spp. as intestinal pathogens comes, in part, from reports of a high rate of faecal carriage of aeromonads in asymptomatic individuals (Pitarangsi et al., 1982). Failure to observe an association between isolation of faecal aeromonads and diarrhoea is more likely when very sensitive methods are used for examination of stool specimens. Enrichment allows recovery of bacteria shed in low numbers; for example, enrichment enables the isolation of Vibrio cholerae from convalescent patients and from patients with subclinical infection as well as from carriers (Rennels et al., 1980). Thus, if enrichment were used routinely,

Received 30 Jul. 1985; accepted 15 May 1986. many faecal isolates of $V$. cholerae would appear to be not associated with diarrhoea at the time of sampling.

\section{Materials and methods}

\section{Faecal specimens}

During a period of 6 weeks in late autumn and early winter, 1981, all faecal samples from patients with diarrhoea and from matched controls (Gracey et al., 1982) submitted to the Department of Microbiology, Princess Margaret Hospital, Perth, were cultured in parallel by direct plating and with enrichment.

In this epidemiological study, patients were considered to have diarrhoea if they had had at least three loose stools per day within the previous 2 days. Patients were regarded as having no diarrhoea if stools had been normal for 2 weeks before the faecal sample was collected.

\section{Isolation media}

Blood agar containing paranitrophenyl glycerine (PNPG) $43 \mu \mathrm{g} / \mathrm{ml} \mathrm{w} / \mathrm{v}$ was used as the medium for primary plating. Nutrient broth (Cowan, 1974) and alkaline peptone water (Furniss et al., 1978) were used for enrichment; they were held at room temperatures for $24 \mathrm{~h}$ and then plated on to the blood-agar PNPG medium.

\section{Identification}

Haemolytic and non-haemolytic colonies were tested 
Table. Association between presence or absence of diarrhoea and method of isolation of Aeromonas spp.

\begin{tabular}{lrrrrrrr}
\multicolumn{8}{c}{ Number of Aeromonas isolates } \\
Method of isolation & $\mathrm{D}^{+}$ & $\mathrm{D}$ & $\mathrm{D}^{+}$ & $\mathrm{D}^{-}$ & $\mathrm{D}^{+}$ & $\mathrm{D}^{-}$ \\
Primary plating & 21 & 3 & 4 & $1^{*}$ & $2^{*}$ & $7^{*}$ \\
Enrichment & 7 & 28 & 2 & 6 & $\mathrm{I}^{*}$ & $3^{*}$
\end{tabular}

$D^{+}=$from patient with diarrhoea; $D=$ from subject with no diarrhoea

* Non enterotoxigenic strains

for oxidase production (Kovacs, 1956), and oxidasepositive colonies were further identified by subculture in the medium of Kaper et al. (1979). Aeromonas spp. were classified according to the methods of Popoff (1984) as $A$. sobria. A. hydrophila or A. caviae. Enterotoxigenic strains were identified by the suckling mouse test as described previously (Burke et al., 1981).

\section{Results}

During the 6 weeks of the study, 38 strains of Aeromonas spp. were isolated from primary plates and also after enrichment; 47 strains were isolated after enrichment but not from primary plates.

The table shows the number of strains and species of Aeromonas isolated by the two methods in relation to the presence of diarrhoea. Of the 43 enterotoxigenic strains isolated only after enrichment, 34 were not associated with diarrhoea. Only three of 28 enterotoxigenic Aeromonas strains isolated on primary plating were not associated with diarrhoea at the time of sampling and two of these were from patients who had a history of diarrhoea within the preceding month. Nine patients from whom enterotoxigenic Aeromonas spp. were isolated only after enrichment had diarrhoea; six strains were isolated from patients with diarrhoea of more than 2 weeks' duration. A shigella was isolated from one sample that also contained $A$. sobria.

\section{Discussion}

Rennels et al. (1980) compared isolation of $V$.

\section{REFERENCES}

Burke V. Robinson J. Berry R J. Gracey M 1981 Detection of enterotoxins of Aeromonas hydrophila by a suckling mouse test. Journal of Medical Microhiology 14:401-408. cholerae by direct plating with isolation after enrichment and found that in $96 \%$ of acute diarrhoeal stools that contained $V$. cholerae, the organism was detected by direct plating. In contrast, vibrios were isolated by direct plating from only $66 \%$ of formed stools that were shown to contain $V$. cholerae by enrichment. Our experience suggests that there is a similar correlation between diarrhoea and the isolation of enterotoxigenic Aeromonas spp. by direct plating; $89 \%$ of strains isolated by direct plating were associated with acute diarrhoea and $7 \%$ with diarrhoea within the preceding month. Of the nine patients with diarrhoea from whom enterotoxigenic Aeromonas spp. were isolated only after enrichment, only two had acute diarrhoea not known to be associated with another intestinal pathogen; $79 \%$ of enterotoxigenic Aeromonas spp. isolated only after enrichment were not associated with diarrhoea within 2 weeks of collecting the faecal sample. We have found that Aeromonas spp. may continue to be excreted in low concentrations by convalescent patients 2 months after symptoms have disappeared.

The data suggest that, as with $V$. cholerae (Rennels et al., 1980), enrichment allows detection of faecal aeromonads present in low concentration that may be found in convalescent patients, carriers, and in cases of subclinical infection with no diarrhoea at the time of sampling. On this basis, we recommend that, as for $V$. cholerae (Gangarosa et al., 1968; Rennels et al., 1980), stools from patients with acute diarrhoea should be plated directly on media for the isolation of Aeromonas spp., without enrichment, but that enrichment should be considered for specimens from convalescent patients, possible carriers, and from those with mild or chronic diarrhoea. Alkaline peptone water or nutrient broth are satisfactory enrichment media for Aeromonas spp.

Epidemiological studies in which enrichment cultures are used but in which the results of Aeromonas spp. isolated only after enrichment are not separated from those isolated by direct plating, are likely to obscure any relationship between diarrhoeal disease and faecal Aeromonas spp.

This work was supported by the TVW Telethon Foundation.

Cowan S T (ed) 1974 Cowan and Steel's Manual for identification of medical bacteria. The Cambridge University Press, Cambridge.

Echeverria P, Blacklow N R. Sanford, L B, Cukor G G 1981 Traveler's diarrhea among American peace corps volun- 
teers in rural Thailand. Journal of Infectious Diseases 143:767-771.

Furniss A L, Lee J V, Donovan T J 1978 The Vibrios. Public Health Laboratory Service Monograph Series No. 11. Her Majesty's Stationery Office, London.

Gangarosa E J, Dewitt W E, Huq I, Zarifi A 1968 Laboratory methods in cholera: isolation of Vibrio cholerae (El Tor and classical) on TCBS medium in minimally equipped laboratories. Transactions of the Royal Society of Tropical Medicine and Hygiene 62:693-699.

Gracey M, Burke V, Robinson J 1982 Aeromonas-associated gastroenteritis. Lancet 2:1304-1306.

Kaper J, Seidler R J, Lockman H, Colwell R R 1979 Medium for the presumptive identification of Aeromonas hydrophila and Enterobacteriacae. Applied and Environmental Microbiology 38:1023-1026.

Kovacs N 1956 Identification of Pseudomonas pyocyanea by the oxidase reaction. Nature 178: 703 .

Millership S E, Chattopadhyay B 1984 Methods for the isolation of Aeromonas hydrophila and Plesiomonas shigelloides from faeces. Journal of Hygiene 92:145-152.

Millership S E, Curnow S R and Chattopadhyay B 1983 Faecal carriage rate of Aeromonas hydrophila. Journal of Clinical Pathology 36:920-923.

Moulsdale M T 1983 Isolation of Aeromonas from faeces. Lancet 1:351.

Pitarangsi C et al. 1982. Enteropathogenicity of Aeromonas hydrophila and Plesiomonas shigelloides: prevalence among individuals with and without diarrhea in Thailand. Infection and Immunity 35:666-673.

Popoff M 1984 Aeromonas Kluyver and Van Niel. In: Krieg N R, Holt J G (eds) Bergey's Manual of systematic bacteriology, 9 th edn, vol. 1. Williams and Wilkins Co., Baltimore, pp 545-548.

Price E H, Hunt G H, Patel U, Walker-Smith J A 1984 Aeromonas spp. in diarrhoea. British Medical Journal 289:1380.

Rennels M B, Levine M M, Daya V, Angle P, Young C 1980 Selective vs. non selective media and direct plating vs enrichment technique in isolation of Vibrio cholerae: recommendations for clinical laboratories. Journal of Infectious Diseases 142:328-331.

Robinson J, Burke V, Worthy P J, Beaman J, Wagener L 1984 Media for isolation of Aeromonas spp. from faeces. Journal of Medical Microbiology 18:405-411.

Rogol M, Sechter I, Grinberg L, Gerichter C B 1979 Pril-xyloseampicillin agar, a new selective medium for the isolation of Aeromonas hydrophila. Journal of Medical Microbiology 12:229-231.

Shread P, Donovan T J, Lee J V 1981 A survey of the incidence of Aeromonas in human faeces. Society for General Microbiology Quarterly 8:184.

Von Graevenitz A, Bucher C 1983 Evaluation of differential and selective media for isolation of Aeromonas and Plesiomonas spp. from human feces. Journal of Clinical Microbiology 17:16-21. 